\title{
Exploration of Technical Debt in Start-ups
}

\author{
Eriks Klotins ${ }^{*}$ \\ Blekinge Institute of Technology \\ Karlskrona, Sweden \\ eriks.klotins@bth.se \\ Tony Gorschek \\ Blekinge Institute of Technology \\ Karlskrona, Sweden
}

\author{
Michael Unterkalmsteiner \\ Blekinge Institute of Technology \\ Karlskrona, Sweden
}

\author{
Rafael Prikladnicki \\ Pontifical Catholic University of Rio \\ Grande do Sul \\ Porto Alegre, Brazil \\ Leandro Bento Pompermaier \\ Pontifical Catholic University of Rio \\ Grande do Sul \\ Porto Alegre, Brazil
}

\author{
Panagiota Chatzipetrou \\ Blekinge Institute of Technology \\ Karlskrona, Sweden
}

\author{
Nirnaya Tripathi \\ University of Oulu \\ Oulu, Finland
}

\begin{abstract}
Context: Software start-ups are young companies aiming to build and market software-intensive products fast with little resources. Aiming to accelerate time-to-market, start-ups often opt for ad-hoc engineering practices, make shortcuts in product engineering, and accumulate technical debt.

Objective: In this paper we explore to what extent precedents, dimensions and outcomes associated with technical debt are prevalent in start-ups.

Method: We apply a case survey method to identify aspects of technical debt and contextual information characterizing the engineering context in start-ups.

Results: By analyzing responses from 86 start-up cases we found that start-ups accumulate most technical debt in the testing dimension, despite attempts to automate testing. Furthermore, we found that start-up team size and experience is a leading precedent for accumulating technical debt: larger teams face more challenges in keeping the debt under control.

Conclusions: This study highlights the necessity to monitor levels of technical debt and to preemptively introduce practices to keep the debt under control. Adding more people to an already difficult to maintain product could amplify other precedents, such as resource shortages, communication issues and negatively affect decisions pertaining to the use of good engineering practices.
\end{abstract}

\section{KEYWORDS}

Software start-ups, technical debt

\footnotetext{
${ }^{*}$ Corresponding author
}

\section{Reference Format:}

Eriks Klotins, Michael Unterkalmsteiner, Panagiota Chatzipetrou, Tony Gorschek, Rafael Prikladnicki, Nirnaya Tripathi, and Leandro Bento Pompermaier. 2018. Exploration of Technical Debt in Start-ups. In ICSESEIP '18: 40th International Conference on Software Engineering: Software Engineering in Practice Track, May 27-fune 3, 2018, Gothenburg, Sweden.

\section{INTRODUCTION}

Start-ups are important suppliers of innovation, new software products and services. However, engineering the software in start-ups is a complex endeavor as the start-up context poses unique challenges to software engineers [10]. As a result of these challenges, most start-ups do not survive the first few years of operation and cease to exist before delivering any value $[4,11]$.

Uncertainty, changing goals, limited human resources, extreme time and resource constraints are reported as characteristic to startups [10, 27]. To cope with such forces, start-ups make a trade-off between internal product quality and faster time-to-market, favoring the latter. As a consequence, start-ups accumulate technical debt [5].

Technical debt is a metaphor to describe not quite right engineering solutions in a product that adds friction to its further development and maintenance. The extra effort associated with this friction, i.e. the "interest", needs to be paid every time a sub-optimal solution is touched [21]. Over time, the cumulative interest may exceed the effort needed to remove the debt, i.e. the "principal". The compound effects of sub-optimal solutions can reduce development team efficiency and overall quality. However, there is the belief among start-ups that any amount of technical debt can be written off if a feature or the whole product is not successful in the market [5].

The strategy to accumulate technical debt can backfire if a startup survives long enough and fails to put its technical debt under control. An unstable and difficult to maintain product adds risk to the company, for example, by limiting the ability to quickly enter into new markets (i.e. to pivot [38]) or to launch new innovative features $[19,39]$. That said, we are not advocating for the removal of all technical debt. Rather, we are interested to see an overview 
of how technical debt influences start-ups and to enable start-up teams to make better decisions in regards to the trade-off between quality and time-to-market.

Technical debt has been extensively studied in the context of established companies and in relation to software maintenance [23, 34]. For example, Tom et al. [39] present a taxonomy comprising precedents, dimensions, and outcomes of technical debt. We adopt the terminology from this taxonomy to enable traceability.

Precedents are contextual factors in the development organization that contribute to the accumulation of technical debt, e.g. a lack of resources. Dimensions describe different types of technical debt, e.g. documentation, architecture, or testing debt. Outcomes refer to consequences of having excess technical debt, such as impaired productivity or quality [39].

While technical debt is a liability, development teams should manage it and use it as a leverage to attain otherwise unattainable goals [39]. In the start-up context, the concept of technical debt is explored only superficially. Giardino et al. [5] argue that the need for speed, cutting edge technologies and uncertainty about a product's market potential are the main precedents for cutting corners in product engineering. However, if a start-up survives past its initial phases, management of technical debt becomes more and more important [5, 7].

Our earlier study on software engineering anti-patterns in startups [19] indicates that poorly managed technical debt could be one contributing factor to high start-up failure rates, driven by poor product quality and difficult maintenance. Negative effects of technical debt on team productivity has also been observed [5].

In this study, we explore how start-ups estimate technical debt, what are precedents for accumulating technical debt, and to what extent start-ups experience outcomes associated with technical debt. We use a case survey as data source and apply a combination of quantitative and qualitative methods to explore technical debt in the surveyed companies. Our objective is to provide a fine-grained understanding of technical debt and its components that could provide a basis for defining start-up context-specific practices for technical debt management.

The main contribution of this paper is an empirical investigation that identifies the key precedents for the accumulation of technical debt in software start-ups, and the primary dimensions where the accumulation of debt has been observed by practitioners.

The rest of the paper is structured as follows. In Section 2 we introduce relevant concepts to understand our study. Section 3 presents the study design while results are presented in Section 4. The results are discussed and interpreted in Section 5. Section 6 concludes the paper.

\section{BACKGROUND AND RELATED WORK}

\subsection{Software start-ups}

Software start-ups are small companies created for the purpose of developing and bringing an innovative product or service to market, and to benefit from economy of scale. Even though start-ups share many characteristics with small and medium enterprises, start-ups are different due to the combination of challenges they face [25, 37].

Start-ups are characterized by high risk, uncertainty, lack of resources, rapid evolution, immature teams, and time pressure among other factors. However, start-ups are flexible to adopt new engineering practices, and reactive to keep up with emerging technologies and markets [11, 37].

Start-up companies rely on external funding to support their endeavors. In 2015 alone, start-up companies have received investments of 429 billion USD in the US and Europe alone [29, 30]. With an optimistic start-up failure rate of $75 \%$ that constitutes of 322 billion USD of capital potentially wasted on building unsuccessful products.

Earlier studies show that product engineering challenges and inadequacies in applied engineering practices could be linked to start-up failures $[10,19]$. To what extent software engineering practices are responsible or linked to success rate is very hard to judge. However, if improved software engineering practices could increase the likelihood of success by only a few percent, it would yield a significant impact on capital return.

\subsection{Technical debt}

Technical debt is a metaphor to describe the extra effort arising from maintaining and removing suboptimal or flawed solutions from a software product. Technical debt can be attributed to the software itself (e.g. source code), and also other artifacts and processes that comprise the product, and are relevant for maintenance and evolution of the product. For example, user manuals, knowledge distribution, operational processes, and infrastructure [39].

Suboptimal solutions find their way into software products due to a variety of reasons, such as ignorance of good engineering practices, oversight, lack of skills, or pragmatism [2]. Taking engineering shortcuts and delivering flawed solutions is often used as leverage to achieve faster time-to-market. However, the debt should be re-payed by removing flawed solutions from the product $[23,39]$.

When not addressed, suboptimal solutions make maintenance and evolution of software products difficult, any changes in the product require more effort than without the debt. This extra effort takes time away from developing new features and may overwhelm a team with firefighting tasks just to keep the product running, and decreases product quality altogether [21].

Giardino et al. [5] argue that technical debt in start-ups accumulates from prioritizing development speed over quality, team aspects, and lack of resources. We combine results from their work, which is specific to start-ups, with a general taxonomy of technical debt by Tom et al. [39]. We adopt the model of precedents, dimensions, and outcomes as proposed by Tom et al. [39] and map it with the categories of the Greenfield start-up model [5] to identify and to focus on relevant aspects of technical debt for start-ups, see Fig. 1.

As precedents, we study engineering skills and attitudes, communication issues, pragmatism, process, and resources. We explore technical debt in forms of code smells, software architecture, documentation, and testing. Furthermore, we attempt to understand to what extent team productivity and product quality is a challenge in start-ups. We use this conceptual model of technical debt as a basis to scope and define the research methodology, discussed next. 


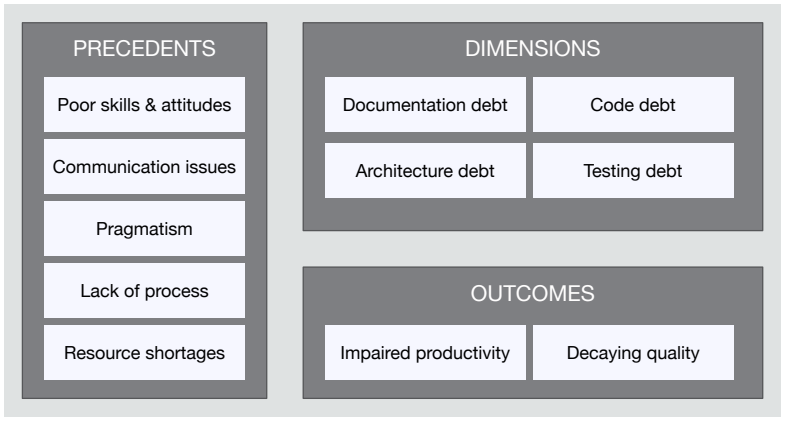

Figure 1: Aspects of technical debt

\section{RESEARCH METHODOLOGY}

\subsection{Research questions}

To achieve our goal and to drive the study we formulate the following research questions:

RQ1: How do start-ups estimate technical debt?

Rationale: Technical debt can incur in different forms, for example, as code smells, incomplete or outdated documentation, suboptimal software architecture, or shortcuts taken in testing [23]. We aim to understand how start-ups estimate different types of technical debt, what types of technical debt are prevalent in start-ups and primary candidates for further investigation. In addition, what types of technical debt are least accumulated, i.e. are irrelevant or already well managed in the start-up context.

RQ2: What are precedents of technical debt in start-ups? Rationale: Earlier studies report a number of precedents contributing to the accumulation of technical debt, such as prioritizing timeto-market over product quality and severe lack of resources [5], developer skills and attitude, lack of process, oversight, and ignorance [39]. We aim to corroborate what precedents, identified by earlier studies in other contexts, are also present in start-ups.

RQ3: What outcomes linked to technical debt do start-ups report? Rationale: Decreasing productivity, decaying morale, product quality issues, and increasing risks are reported as outcomes of technical debt $[5,39]$. Yet, there is a belief that any amount of technical debt can be written off if a product or a specific feature does not succeed in market [5]. We aim to corroborate what outcomes, identified by earlier studies and linked to increased amounts of technical debt, do start-ups report.

\subsection{Data collection}

We used a case survey method to collect primary data from start-up companies [22,28].

The case survey method is based on a questionnaire and is a compromise between a traditional case study and a regular survey [17]. We have designed the questionnaire to collect practitioners experiences about specific start-up cases.

During the questionnaire design phase, we conducted multiple internal and external reviews to ensure that all questions are relevant, clear and that we receive meaningful answers. First, the questions were reviewed in multiple rounds by the first three authors of this paper to refine scope of the survey and question formulations. Then, with help of other researchers from the Software Start-up Research Network $^{1}$, we conducted a workshop to gain external input on the questionnaire. A total of 10 researchers participated and provided their input.

Finally, the questionnaire was piloted with four practitioners from different start-ups. During the pilots, respondents filled in the questionnaire while discussing questions, their answers and any issues with the first author of this paper.

As a result of these reviews, we improved the question formulations and removed some irrelevant questions. The finalized questionnaire ${ }^{2}$ contains 85 questions in 10 sections. The questionnaire captures 285 variables from each start-up case.

From all the variables, 45 variables focus on capturing the magnitude of dimensions, precedents, and outcomes linked to technical $\operatorname{debt}^{3}$. The questions capture the respondents' agreement with a statement on a Likert scale: not at all (1), a little (2), somewhat (3), very much (4). The values indicate the degree of agreement with a statement. Statements are formulated consistently in a way that lower values indicate less precedents, less outcomes, and less technical debt.

In addition to questions pertaining technical debt, the questionnaire contains questions inquiring the engineering context in the start-up and applied software engineering practices.

The data collection took place between December 1, 2016, and June 15,2017 . The survey was promoted through personal contacts, by attending industry events, and by posts on social media websites. Moreover, we invited other researchers from the Software Startup Research Network to collaborate on the data collection. This collaboration helped to spread the survey across many geographical locations in Europe, North and South America, and Asia.

\subsection{Data analysis}

To analyze the survey responses we used a number of techniques. We started by screening the data and filtering out duplicate cases, responses with few questions answered, or otherwise unusable responses. In the screening we attempt to be as inclusive as possible and do not remove any cases based on the provided responses.

The respondent estimates on technical debt aspects are measured on an ordinal scale, measured from 1 (not at all) to 4 (very much). Respondent and start-up demographics such as age and years of operation are measured with categorical variables on a nominal scale.

Overall, we analyze responses from 86 start-up cases, 75 datapoints per each case, and 6450 data-points overall. To gain an overview of the data, the results were visualized by histograms, box-plots and contingency tables [12].

We use the Chi-Squared test of association to test if the associations between the examined variables are not due to chance. To prevent Type I errors, we used exact tests, specifically, the MonteCarlo test of statistical significance based on 10000 sampled tables and assuming $(p=0.05)[14]$.

\footnotetext{
${ }^{1}$ The Software Start-up Research Network, https://softwarestartups.org/

${ }^{2}$ http://startupcontextmap.org/exp-survey/woifenw2

${ }^{3}$ The subset of questions used in this study is available here: http://eriksklotins.lv/ uploads/TD-in-start-ups-questions.pdf
} 
Table 1: Interpretation of Cramer's V test

\begin{tabular}{cl} 
Cramer's V value & Interpretation \\
\hline$\geq 0.1$ & Weak association \\
$\geq 0.3$ & Moderate association \\
$\geq 0.5$ & Strong association \\
\hline
\end{tabular}

To examine the strength of associations we use Cramer's V test. We interpret the test results as suggested by Cohen [6], see Table 1 . To explore specifics of the association, such as which cases are responsible for this association, we perform post-hoc testing using adjusted residuals. We consider an adjusted residual significant if the absolute value is above 1.96 (Adj.residual > 1.96), as suggested by Agresti [1]. The adjusted residuals drive our analysis on how different groups of start-ups estimate aspects of technical debt. However, due to the exploratory nature of our study, we do not state any hypotheses upfront and drive our analysis with the research questions.

Full results, contingency tables, histograms and calculation details are accessible on-line ${ }^{4}$ for a full disclosure.

\subsection{Validity threats}

In this section we follow guidelines by Runeson et al. [32] and discuss four types of validity threats and applied countermeasures in the context of our study.

3.4.1 Construct validity. Construct validity concerns whether operational measures really represent the studied subject [32]. A potential threat is that the statements we use to capture respondent estimates are not actually capturing the studied aspects of technical debt.

To address this threat we organized a series of workshops with other researchers and potential respondents to ensure that questions are clear, to the point, and capture the studied phenomenon.

Each aspect, i.e. type of precedent, is triangulated by capturing it by at least three different questions in the questionnaire. To avoid biases stemming from respondents opinions about technical debt and to capture the actual situation we avoid mentioning technical debt in the questions. Instead, we formulate the questions indirectly to capture respondent estimates on different aspects associated with technical debt. For example, we ask whether they find it difficult to understand requirements documentation.

To accommodate for the fact that a respondent may not know answers to some of the questions, we provide an explicit "I do not know" answer option to all Likert scale questions.

3.4.2 Internal validity. This type of validity threat addresses causal relationships in the study design [32]. In our study we use a model of precedents, dimensions and outcomes of technical debt. The literature, for example, Tom et al. [39] and Li et al. [23], suggest that there is a causality between the three. We, however, present respondent estimates on precedents, dimensions and the outcomes separately without considering or implying any causality.

3.4.3 External validity. This type of validity threat concerns to what extent the results could be valid to start-ups outside the

\footnotetext{
${ }^{4}$ http://eriksklotins.lv/uploads/TD-in-start-ups-sm.pdf
}

study [32]. The study setting for participants was close to real life as possible, that is, the questionnaire was filled in without researcher intervention and in the participants own environment.

A sampling of participants is a concern to external validity. We use convenience sampling to recruit respondents and with help of other researchers, distributed the survey across a number of different start-up communities. Demographic information from respondent answers shows that our sample is skewed towards active companies, respondents with little experience in start-ups, young companies and small development teams of 1-8 engineers. In these aspects our sample fits the general characteristics of startups, see for example, Giardino et al. [10,11] and Klotins et al. [18]. However, there clearly is a survivorship bias, that is, failed start-ups are underrepresented, thus our results reflect state-of-practice in active start-ups.

Another threat to external validity stems from case selection. The questionnaire was marketed to start-ups building softwareintensive products, however due to the broad definition of software start-ups (see Giardino et al. [11]), it is difficult to differentiate between start-ups and small medium enterprises. We opted to be as inclusive as possible and to discuss relevant demographic information along with our findings.

3.4.4 Conclusion validity. This type of validity threat concerns the possibility of incorrect interpretations arising from flaws in, for example, instrumentation, respondent and researcher personal biases, and external influences [32].

To make sure that respondents interpret the questions in the intended way we conducted a number of pilots, workshops and improved the questionnaire afterwards. To minimize the risk of systematic errors, the calculations and statistical analysis was performed by the first and the third author independently, and findings were discussed among the authors.

To strengthen reliability and repeatability of our study, all survey materials and calculations with immediate results are published online.

\section{RESULTS}

To answer our research questions we analyze 6450 data-points from 86 start-up cases. The majority of these start-ups ( 63 out of $86,73 \%$ ) are active and had been operating for 1 - 5 years (58 out of $86,67 \%$ ), see Fig. 2. Start-ups are geographically distributed among Europe (34 out of 86, 40\%), South America (41 out of 86, 47\%), Asia (7 out of 86) and North America (2 out of 86).

Our sample is about equally distributed in terms of the product development phase. We follow a start-up life-cycle model proposed by Crowne [7] and distinguish between inception, stabilization, growth and maturity phases. In our sample, 16 start-ups have been working on a product but haven't yet released it to market, 24 teams had released the first version and actively develop it further with customer input, 26 start-ups have a stable product and they focus on gaining customer base, and another 16 start-ups have mature products and they focus on developing variations of their products. The distribution of start-ups by their life-cycle phase and length of operation is shown in Fig. 3. In the figure, bubble size denotes the a number of people in the team. Most start-ups in the sample (75 out 


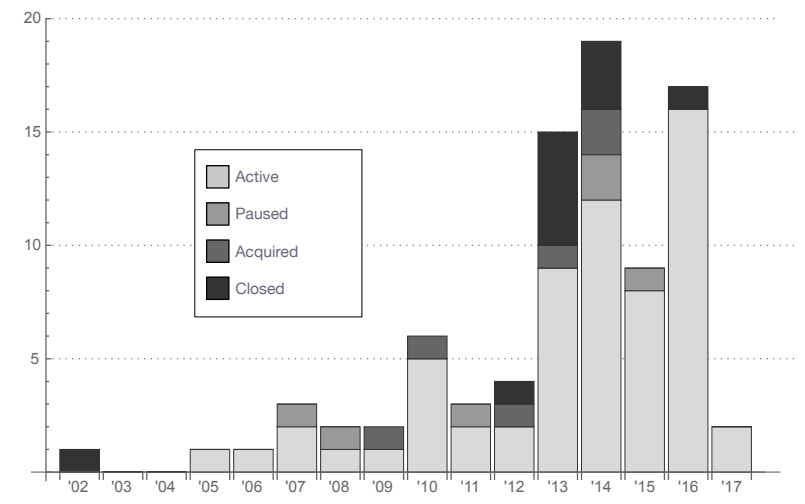

Figure 2: Distribution of start-ups by the founding year and their current state

of $86,87 \%$ ) have small teams of $1-8$ engineers actively working on the product.

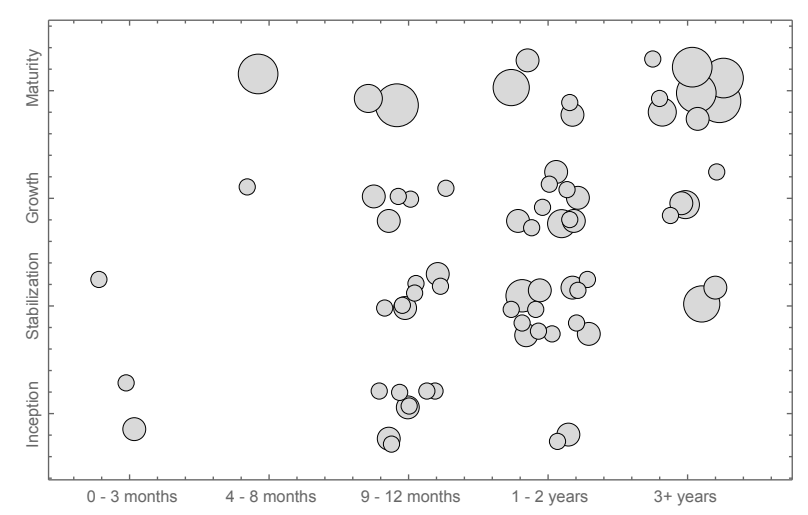

Figure 3: Distribution of start-ups by product phase and length of operation

About an equal number of start-ups had indicated that they work on more than one product at the time. Start-ups in our sample do per-customer customization to some extent: 10 companies (11\%) had specified that they tailor each product instance to a specific customer, 30 companies (35\%) do not do per-customer customization at all, while 43 start-ups (49\%) occasionally perform product customization for an individual customer.

The questionnaire was filled in mostly by start-up founders (64 out of $86,74 \%$ ) and engineers employed by start-ups (15 out of 86, $17 \%$ ). About a half of respondents have specified that their area of expertise is software engineering (49 out of $86,56 \%$ ). Others have specified marketing, their respective domain, and business development as their areas of expertise.

Respondents length of software engineering experience ranges from 6 months to more than 10 years. A large portion of respondents (44 out of 86,51\%) had less than 6 months of experience in working with start-ups at the time when they joined their current start-up.

\subsection{Dimensions of technical debt}

We start our exploration by looking at the extent to which the dimensions of technical debt (documentation, architecture, code, and testing) are present in the surveyed start-ups. We quantify the degree of technical debt by aggregating respondent answers on questions pertaining to each dimension, Answers were given on a Likert scale where higher values indicate more estimated technical debt in a given dimension.

Responses from the whole sample indicate that start-ups estimate some technical debt ( 2 on a scale from 1 to 4 ) in documentation, architecture, and code dimensions, while testing debt is estimated as the most prevalent ( 3 in a scale from 1 to 4 ). Fig. 4 shows the median (dark horizontal line), first and third quartile, and minimum and maximum estimates on all statements pertaining to a specific debt type.

To explore the estimated degree of technical debt further, we analyze the influence of respondent demographics, such as relationship with the start-up and background, and start-up demographics, such as product life-cycle phase, team skill level and longevity of the start-up, on the responses.

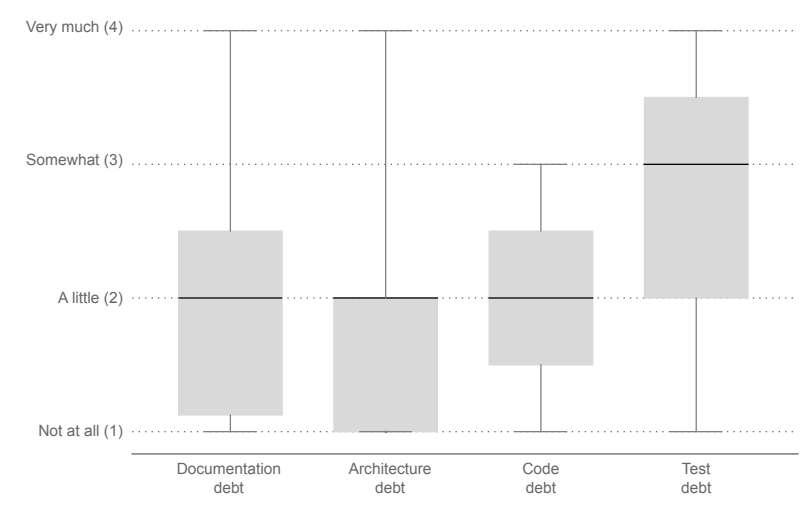

Figure 4: Estimates for the prevalence of different dimensions of technical debt from the case survey

The analysis shows that only start-up state, that is if the start-up is active, paused, acquired, or closed, has an effect on the overall estimates of technical debt, see Table 2 . In the table we show strength (measured by Cramer's V test) of statistically significant associations ( $p \leq 0.05$, measured by Chi-Square test) between relevant characteristics of start-ups and technical debt dimensions. In the last column we show if the characteristic has an effect on all dimensions together.

Observe that the level of engineering skills and domain knowledge pertains to the whole team. However, practical experience pertains only to the respondent. We show respondent characteristics as well to illustrate to what extent respondents background influences their responses. For instance, respondents with more practical experience estimate documentation debt more critically, see Table 2, and are more critical about skills shortages, see Table 3.

We highlight important findings in framed boxes and discuss them in Section 5. 
Table 2: Results of Cramer's V test on associations between dimensions and start-up characteristics with $p \leq 0.05$

\begin{tabular}{lllllll}
$\#$ & Characteristic & Documentation & Architecture & Code & Testing & All \\
\hline 1 & State of the start-up & 0.346 & 0.326 & 0.414 & - & 0.318 \\
2 & Product phase & - & 0.329 & - & - & - \\
3 & Overall team size & - & - & 0.427 & - & - \\
4 & Level of domain knowledge & 0.334 & - & - & - & - \\
5 & Per-customer tailoring & - & - & 0.423 & - & - \\
6 & Practical experience & 0.337 & - & - & - & \\
\hline
\end{tabular}

Finding 1: Start-ups that are in the active category estimate technical debt, overall in all dimensions, lower than closed or acquired start-ups.

Product phase, team size and level of domain knowledge have effects on individual technical debt dimensions. We present these results next.

4.1.1 Documentation debt. Documentation debt refers to any shortcoming in documenting aspects of software development, such as architecture, requirements, and test cases [23].

We look into requirements, architecture and test documentation because these are the essential artifacts guiding a software project. Requirements capture stakeholders needs and provide a joint understanding of what features are expected from the software. Architecture documentation lists design principles, patterns and components comprising the software. Documentation of test cases supports testing activities and provides means for quality assurance [33].

Only $1 \%$ (7 out of 84 ) of start-ups in our sample have explicitly stated that they do not document requirements in any way. The most popular forms of documenting requirements are informal notes and drawings (50 out of $86,58 \%$ ), followed by organized lists (20 out of $86,20 \%$ ).

Responses from the whole sample show that start-ups have some amount of documentation debt (Median $=2.0$ ), see Fig. 4. Exploring what start-up characteristics have an effect on the estimates, see Table 2, we found that start-ups who are active estimate documentation debt lower than acquired or closed companies. We also found that teams with sufficient domain knowledge estimate documentation debt lower than teams with many gaps in their domain knowledge.

4.1.2 Architecture debt. Architecture debt refers to compromises in internal qualities of the software such as maintainability, scalability, and evolvability [23].

Results from the whole sample show that start-ups experience some architectural debt (Median $=2.0$ ), see Fig. 4 . By looking into what start-up characteristics have an effect on how respondents estimate architecture debt, we found that state of the start-up and the product phase have an effect on the estimates, see Table 2. Active start-ups have provided substantially lower estimates than acquired and closed companies. We found that start-ups who have just started on product engineering and haven't yet released it to market, experience almost no architectural debt. During stabilization and growth phases the estimates become more critical. However, during the maturity phase estimates become slightly more optimistic, see Fig. 5. Box-plots in the figure show responses from all statements pertaining to architecture debt.

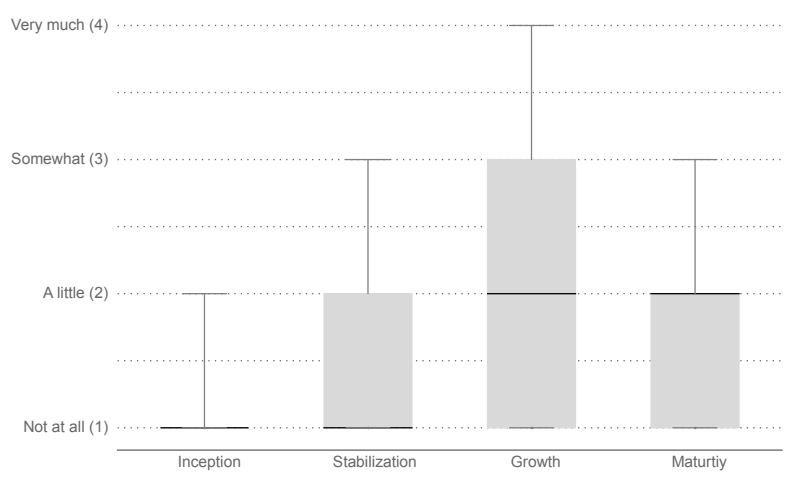

Figure 5: Box-plot showing how in different product phases start-ups estimate architecture debt

4.1.3 Code debt. Code debt refers to a poorly written code. Signs of a poorly written code are, for example, unnecessary complex logic, code clones, and bad coding style affecting code readability. Poorly written code is difficult to understand and change $[24,26$, 39].

Results from the whole sample show that start-ups experience some code debt (Median = 2.0), see Fig. 4. By looking into what start-up characteristics have an effect on how respondents estimate code debt we found that state of the start-up, team size, level of per-customer tailoring has an effect on the estimates, see Table 2. Active start-ups estimate code debt lower than acquired start-ups. Start-ups with larger teams ( 9 or more people), provide higher estimates on code debt than small teams. Start-ups who do not offer per-customer customization estimate code debt lower. However, start-ups that occasionally tailor their product to needs of a specific customer estimates their code debt higher.

4.1.4 Testing debt. Testing debt refers to lack of test automation leading to the need of manually retesting the software before a release. The effort of manual regression testing grows exponentially with the number of features, slowing down release cycles and making defect detection a time consuming and tedious task [39].

Answers to questions inquiring use of automated testing show that about a third (26 out of $86,30 \%$ ) of start-ups are attempting 
to implement automated testing, and only 17 start-ups (20\%) have explicitly stated that no test automation is used.

Despite attempts to automate, companies across the whole sample estimate their testing debt somewhat high (Median $=3$ ), see Fig. 4. Manual exploratory testing is reported as the primary testing practice, regardless of start-up life-cycle phase, team size and engineering experience, and length of operation.

Finding 2: Despite attempts to automate, manual testing is still the primary practice to ensure that the product is defect free.

Similar results, showing that only a small number of mobile application projects have any significant code coverage by automated tests, and listing time constraints as the top challenge for adopting automated testing, were obtained by Kochhar et al. [20].

\subsection{Precedents for technical debt}

We asked the respondents to estimate various precedents of technical debt in their start-ups, such as attitudes towards good software engineering practices, pragmatic decisions to make shortcuts in product engineering, communication issues in the team, level of team engineering skills, time and resource shortages, and lack of established SE processes.

Box-plots with median responses from the whole sample are shown in Fig. 6. Higher values indicate stronger agreement with the presence of a precedent in the start-up. Poor attitude is the least common precedent for technical debt, while resource shortage is estimated as the most prevalent precedent.

Looking into what start-up characteristics influence the responses, we find that start-up team size and team's engineering skills have a significant effect on the estimates overall, see Table 3. Larger teams of 9 or more people estimate the precedents for technical debt higher than small teams.

In the results we show only characteristics with statistically relevant associations, thus listed characteristics differ between Tables 2 and 3.

Finding 3: Start-up team size and level of engineering skills have a significant effect on how severe the other precedents are estimated.

4.2.1 Attitude towards good engineering practices. The responses to questions about following good engineering practices suggest that start-up engineers do realize the importance of following good architecture, coding and testing practices (Median $=1)$, see Fig. 6 .

Comparing how responses on attitude differ by start-up characteristics we found that start-ups who are active, estimate their attitudes more optimistically than acquired or closed companies. That is, they agree more with benefits from following good engineering practices, such as coding conventions and throughout testing of the product.

4.2.2 Pragmatism. Estimates on statements about pragmatic considerations, that is, prioritization of time-to-market over good

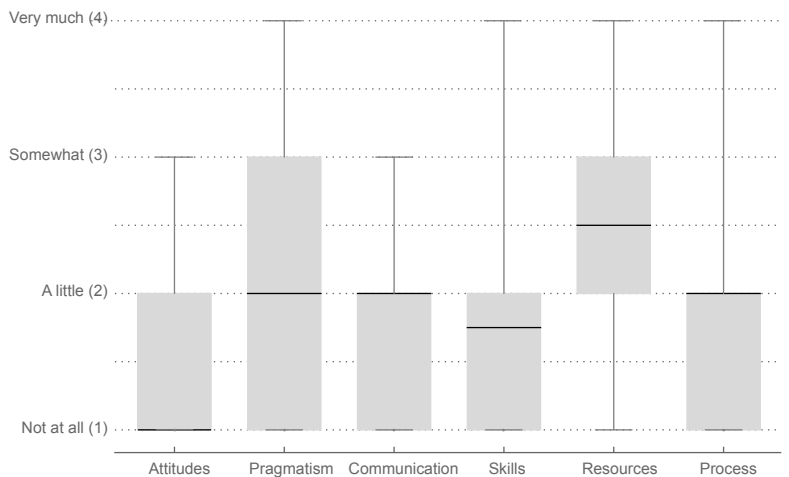

Figure 6: Box-plot showing how the sample estimates different precedents for technical debt

engineering practices, show that start-ups are ready to make shortcuts to speed up time-to-market (Median $=2$ ). However, the spread of estimates suggests that different companies have very different attitudes towards deliberately introducing technical debt, see Fig. 6. Comparing how estimates on attitude differ by start-up characteristics we found that start-ups with larger teams of 15 or more developers estimate pragmatic precedents higher than smaller teams.

4.2.3 Communication. Estimates on statements about communication show that communication issues could be one of the precedents for introducing technical debt, see Fig. 6. We observe that communication issues become significantly more severe in larger engineering teams of 13 and more people than in smaller teams. Moreover, the results suggest that teams with better engineering skills experience fewer communication issues.

4.2.4 Engineering skills. Estimates to what extent start-ups face lack of engineering skills show that skills shortage could be a precedent for accumulating technical debt, see Fig. 6.

Comparing how estimates on skill shortages differ by start-up characteristics we found that the state of the start-up, team size, length of practical experience, and level of estimated engineering skills have the significant influence on the estimates. We found that active start-ups estimate skills shortages lower than closed down companies. A somewhat expected result is that teams with adequate engineering skills provide significantly lower estimates for challenges associated with skills shortages.

4.2.5 Resources. Looking at differences between estimates on time and other types of resources we found that they are tied together, see Fig. 6. That is, companies reporting time shortages also report resource shortages. A potential explanation for the association is that time pressure is created internally by a need to get the product out and start generating revenue, and not by an external market pressure. We also found that per-customer customization, overall team size, and level of domain knowledge has an effect on how start-ups estimate resource shortages.

Estimates of resources and time shortages show that resource issues are the highest estimated precedent for technical debt (Median $=$ 2.5). We find that occasional per-customer tailoring is associated with higher estimates on resource shortages. Potentially, start-ups 
Table 3: Results of Cramer's V test on associations between precedents and start-up characteristics with $p \leq 0.05$

\begin{tabular}{|c|c|c|c|c|c|c|c|c|}
\hline \# & Characteristic & Attitudes & $\begin{array}{l}\text { Pragma- } \\
\text { tism }\end{array}$ & $\begin{array}{l}\text { Communication } \\
\text { issues }\end{array}$ & $\begin{array}{l}\text { Skills } \\
\text { shortages }\end{array}$ & $\begin{array}{l}\text { Resources } \\
\text { shortages }\end{array}$ & Process & All \\
\hline 1 & State of the start-up & 0.339 & - & - & 0.285 & - & - & - \\
\hline 2 & Per-customer tailoring & - & - & - & - & 0.345 & - & - \\
\hline 3 & Overall team size & - & - & - & 0.360 & 0.344 & 0.375 & 0.386 \\
\hline 4 & Development team size & - & 0.432 & 0.387 & 0.429 & - & 0.403 & - \\
\hline 5 & Level of engineering skills & - & - & 0.331 & 0.465 & - & - & 0.377 \\
\hline 6 & Level of domain knowledge & - & - & - & - & 0.324 & - & - \\
\hline 7 & Practical experience & - & - & - & 0.329 & - & - & - \\
\hline
\end{tabular}

suffering from lack of resources opt for occasional customization to serve needs of an important customer, thus acquiring resources for further development. Start-ups with smaller teams of 1-3 people estimate resource shortages lower than larger start-ups of 9-12 people. A plausible explanation for this association could be that supporting a larger team requires more resources.

4.2.6 Process. Respondent estimates on the software engineering process issues show that frequent and unplanned changes occur and could cause difficulties in avoiding technical debt, see Fig. 6 . We found that estimates on process issues become more severe as team size grows.

\subsection{Outcomes of technical debt}

To explore potential outcomes of technical debt we presented respondents with statements exploring to what extent team productivity and product quality are concerns in their start-ups. Estimates from the whole sample show that start-ups experience some quality and productivity issues (Median $=2)$ that could be associated with accumulated technical debt. We found that team size is the only characteristic that influences the estimates $\left(\right.$ Cramer' $\left.^{\prime} \mathrm{s} V=0.362\right)$.

Looking into what types of technical debt are associated with specific outcomes linked to technical debt, we found a clear association between estimates of technical debt and estimates of the outcomes, see Table 4.

Code debt has the most severe impact on both productivity and quality. Architecture debt have a similar effect, albeit to a lesser extent. Documentation debt impairs productivity. However, we did not find a statistically significant association between testing debt and loss of productivity or quality.

Finding 4: We found that from all types of technical debt, code debt have the strongest association with productivity and quality issues.

\section{DISCUSSION}

\subsection{Reflections on the research questions}

Our results on how start-ups estimate technical debt show that active start-ups estimate aspects of technical debt significantly lower than closed or acquired start-ups, see Finding 1 in Section 4.1. A plausible explanation for this result could be that lower technical debt helps start-ups to have a more stable and easier to maintain
Table 4: Results of Cramer's $V$ test on associations between types of technical debt and outcomes with $p \leq 0.05$

\begin{tabular}{lllll}
$\#$ & Debt type & \multicolumn{3}{c}{ Impact on: } \\
& & Quality & Productivity & Both \\
\hline 1 & Documentation & - & 0.332 & 0.344 \\
2 & Architecture & 0.399 & 0.331 & 0.440 \\
3 & Code & 0.445 & 0.471 & 0.532 \\
4 & Testing & - & - & - \\
\hline & All types & 0.363 & 0.459 & 0.463
\end{tabular}

product. Thus giving a start-up more room for evolving the product into something the market wants, i.e. to pivot [3]. However, excess technical debt hinders product evolution and could be one of contributing factors to the shutdown of a company.

An alternative explanation is that technical debt could be invisible and compensated by the team's implicit knowledge. However, when a start-up is acquired by another company and the product is transferred to another team, all the technical debt becomes visible. Difficulties to capture undocumented knowledge and the associated drop in performance of the receiving team has been recognized in the context of agile project handover [36].

Results on how different types of technical debt are estimated show that the most technical debt is estimated in the testing category, even though start-ups do attempt to automate tests, see Finding 2 in Section 4.1. A potential explanation could be that startups lack certain prerequisites for full implementation of automated testing [40]. Excess technical debt in other categories, for instance, difficult to test code, lack of requirements documentation, and an unclear return of investment, could be hindering the implementation of test automation, as it is also observed in traditional, more mature companies [16, 20,31]. However, we could not find any statistically significant association between testing debt and quality or productivity issues.

The comparison of results on architecture debt from start-ups in different life-cycle phases shows another interesting pattern. Startups who have not yet released their products to market experience very little architecture debt, the debt increases as the product is delivered to the first customer and peaks at the growth stage when start-ups focus on marketing the product and drops slightly as start-ups mature, see Fig 5. Marketing of the product could be a source of new challenges for the product development team. For 
example, the product must support different configurations for different customer segments, provide a level of service, and cope with a flow of requests for unanticipated features [7, 8]. Earlier shortcuts in product architecture are therefore exposed and must be addressed.

Overall team size and level of engineering skills could be the most important characteristics contributing to precedents and linked to technical debt in most dimensions, see Finding 3 in Section 4.2 . Larger teams of 9 or more people experience more challenges and report higher technical debt in all categories. This finding is similar to Melo et al. [9] studying productivity in agile teams. Smaller teams are better aligned and more efficient in collaboration with little overhead. However, as the team size grows more processes and artifacts for coordination are needed [35]. Therefore larger teams have more artifacts that can degrade.

Team size could be an indicator of the general complexity of a start-up and the product. More people are added to the team when there are more things to be taken care of. Therefore, the technical debt could stem not only from the number of people but also from increasing complexity of the organization itself.

Our results show that increase in team size is also associated with outcomes of technical debt, a decrease in productivity and product quality, see Finding 4 in Section 4.3. This result could be explained by our earlier discussion on how larger teams require more coordination for collaboration. However, the more critical estimates by larger teams could be also associated with the increase in product complexity as new features are added. Rushing to release new features could contribute to the accumulation of technical debt until deliberate, corrective actions are taken, as observed in mobile application development [13].

As a software product grows, it naturally becomes more difficult to maintain. For instance, if individual product components do not change and the new components are at the same quality level as existing ones, the increased number of components and their dependencies requires more effort from engineers to maintain the product and creates more room for defects [15]. This is software decay and is not the same as avoidable technical debt stemming from the trade-off between quality and speed. Distinguishing between true technical debt and software decay is an important next step in providing practical support for software-intensive product engineering in start-ups.

\subsection{Implications for practitioners}

This study presents several implications for practitioners:

(1) Start-up teams with higher level of engineering skills and respondents with more experience perceive aspects of technical debt more severely. Less skilled teams may not be aware of their practices introducing additional technical debt, and amount of technical debt in their products. Using tools and occasional external expert help could help to identify unrealized technical debt, and to improve any sub-optimal practices.

(2) Start-up team size correlates with more severe precedents and outcomes of technical debt. Keeping a team small and skilled could be a strategy to mitigate precedents for technical debt. To support growth of the team, more coordination practices need to be introduced, and impact on technical debt monitored. Additional coordination practices require more maintenance of coordination artifacts. Thus, there is a practical limit how large a team can grow before it needs to be divided into sub-teams.

(3) There is an association between levels of technical debt and a start-up outcome. Having less technical debt could give a start-up more room for pivoting and product evolution in the long term.

(4) There are certain moments when the effects of technical debt are the most severe. For example, shipping a product to a large number of customers, scaling up the team, and handing the product over to another team. The anticipation of such moments and adequate preparations could help to mitigate the negative effects of technical debt.

(5) The most significant type of technical debt in start-ups is code smells. We found that poorly structured and documented code has the strongest association with issues in team productivity and product quality. However, detection of code smells can be automated with open-source tools, thus alleviating removal of this type of debt.

\section{CONCLUSIONS AND FUTURE WORK}

In this paper, we report how technical debt is estimated in start-ups building software-intensive products. We explore to what extent precedents, dimensions, and outcomes, identified by earlier studies, are relevant in the start-up context. We attempt to identify what start-up characteristics have an amplifying or remedying effect on technical debt.

Our results show that, even though start-up engineers realize the importance of good engineering practices, they cut corners in product engineering, mostly due to resource pressure and a need for faster time to market. The results suggest that precedents for technical debt become more severe as start-ups evolve and severity of the precedents could be associated with the number of people working in a start-up and a product life-cycle phase.

Our results show significantly different estimates from closed, acquired and operational start-ups. The differences highlight how start-ups use technical debt as a leverage, and emphasizes the importance of careful technical debt management.

This exploratory study leads to a formulation of several hypotheses:

(a) Technical debt peaks at the growth stage when a start-up attempts to market the product.

(b) The number of people in a team amplifies precedents for technical debt.

(c) There is an association between a start-up outcome and their technical debt management strategy.

We aim to explore these hypotheses further by triangulating results from this study with qualitative data from interviews and artifact analysis.

\section{ACKNOWLEDGMENTS}

The authors of this paper would like to thank all practitioners who found time and motivation share their experiences. Reaching this diverse population of start-ups would not be possible without help 
and support from Software Start-up Research Network community. and specifically Nana Assyne, Anh Nguyen Duc, Ronald Jabangwe, Jorge Melegati, Bajwa Sohaib Shahid, Xiaofeng Wang, Rafael Matone Chanin, and Pekka Abrahamsson.

\section{REFERENCES}

[1] Alan Agresti. 1996. An introduction to categorical data analysis. Vol. 135. Wiley New York.

[2] Nicolli SR Alves, Thiago S Mendes, Manoel G de Mendonça, Rodrigo O Spínola Forrest Shull, and Carolyn Seaman. 2016. Identification and management of technical debt: A systematic mapping study. Information and Software Technology 70 (2016), 100-121.

[3] Sohaib Shahid Bajwa, Xiaofeng Wang, Anh Nguven Duc, and Pekka Abrahamsson 2016. How do software startups pivot? empirical results from a multiple case study. In International Conference of Software Business. Springer, 169-176.

[4] Steve Blank. 2013. Why the Lean Start Up Changes Everything. Harvard Business Review 91, 5 (2013), 64.

[5] Giardino Carmine, Nicolò Paternoster, Michael Unterkalmsteiner, Tony Gorschek, and Pekka Abrahamsson. 2016. Software Development in Startup Companies: The Greenfield Startup Model. IEEE Transactions on Software Engineering X, September (2016), 233.

[6] J Cohan. 1988. Statistical power analysis for the behaviour sciences. (1988)

[7] Mark Crowne. 2002. Why software product startups fail and what to do about it In Engineering Management Conference. IEEE, Cambridge, UK, 338-343.

[8] Åsa G Dahlstedt, Lena Karlsson, Anne Persson, J Natt och Dag, and Björn Regnell. 2003. Market-Driven Requirements Engineering Processes for Software Products - a Report on Current Practices. In International Workshop on COTS and Product Software, RECOTS 2003

[9] Claudia O. De Melo, Daniela S. Cruzes, Fabio Kon, and Reidar Conradi. 2013. Interpretative case studies on agile team productivity and management. Information and Software Technology 55, 2 (2013), 412-427.

[10] Carmine Giardino, Sohaib Shahid Bajwa, and Xiaofeng Wang. 2015. Key Challenges in Early-Stage Software Startups. In Agile Processes, in Software Engineering, and Extreme Programming, Vol. 212. 52-63.

[11] Carmine Giardino, Michael Unterkalmsteiner, Nicolo Paternoster, Tony Gorschek, and Pekka Abrahamsson. 2014. What Do We Know about Software Development in Startups? IEEE Software 31, 5 (sep 2014), 28-32.

[12] Shelby J Haberman. 1973. The analysis of residuals in cross-classified tables. Biometrics (1973), 205-220.

[13] Geoffrey Hecht, Omar Benomar, Romain Rouvoy, Naouel Moha, and Laurence Duchien. 2015. Tracking the Software Quality of Android Applications Along Their Evolution (T). In Automated Software Engineering (ASE), 2015 30th IEEE/ACM International Conference on. IEEE, 236-247.

[14] Adery CA Hope. 1968. A simplified Monte Carlo significance test procedure Journal of the Royal Statistical Society. Series B (Methodological) (1968), 582-598.

[15] Clemente Izurieta and James M Bieman. 2013. and rot in evolving software systems. (2013), 289-323. https://doi.org/10.1007/s11219-012-9175-x

[16] Mona Erfani Joorabchi, Ali Mesbah, and Philippe Kruchten. 2013. Real challenges in mobile app development. In Empirical Software Engineering and Measurement, 2013 ACM/IEEE International Symposium on. IEEE, 15-24.

[17] Eriks Klotins. 2017. Using the case survey method to explore engineering practices in software start-ups. In Proceedings of the 1st International Workshop on Software Engineering for Startups. IEEE Press, 24-26.

[18] Eriks Klotins, Michael Unterkalmsteiner, and Tony Gorschek. 2015. Software Engineering in Start-up companies : an Exploratory Study of 88 Start-ups. 13, 9 (2015), 1-19.

[19] E Klotins, M Unterkalmsteiner, and T Gorschek. 2017. Software Engineering Anti-patterns in start-ups. In review by IEEE Software (2017).

[20] Pavneet Singh Kochhar, Ferdian Thung, Nachiappan Nagappan, Thomas Zimmermann, and David Lo. 2015. Understanding the test automation culture of app developers. In Software Testing, Verification and Validation (ICST), 2015 IEEE 8th International Conference on. IEEE, 1-10.

[21] Philippe Kruchten, Robert L. Nord, and Ipek Ozkaya. 2012. Technical Debt : From Metaphor. IEEE Software (2012), 18-22.

[22] R. Larsson. 1993. Case Survey Methodology: Quantitative Analysis of Patterns Across Case Studies. Academy of Management fournal 36, 6 (1993), 1515-1546.

[23] Zengyang Li, Paris Avgeriou, and Peng Liang. 2015. A systematic mapping study on technical debt and its management. Fournal of Systems and Software 101 (2015), 193-220.

[24] Mika Mantyla, Jari Vanhanen, and Casper Lassenius. 2003. A taxonomy and an initial empirical study of bad smells in code. International Conference on Software Maintenance, 2003. ICSM 2003. Proceedings. OCTOBER (2003), 381-384.

[25] Andreas Metzger and Klaus Pohl. 2014. Software product line engineering and variability management: achievements and challenges. In Proceedings of the on Future of Software Engineering. ACM, 70-84.
[26] Fabio Palomba, Gabriele Bavota, Massimiliano Di Penta, Rocco Oliveto, and Andrea De Lucia. 2014. Do they really smell bad? A study on developers' perception of bad code smells. Proceedings - 30th International Conference on Software Maintenance and Evolution, ICSME 2014 November 2016 (2014), 101-110.

[27] Nicolò Paternoster, Carmine Giardino, Michael Unterkalmsteiner, Tony Gorschek, and Pekka Abrahamsson. 2014. Software development in startup companies: A systematic mapping study. Information and Software Technology 56, 10 (oct 2014), $1200-1218$.

[28] Kai Petersen, Deepika Badampudi, Syed Shah, Krzysztof Wnuk, Tony Gorschek, Efi Papatheocharous, Jakob Axelsson, Severine Sentilles, Ivica Crnkovic, and Antonio Cicchetti. 2017. Choosing Component Origins for Software Intensive Systems: In-house, COTS, OSS or Outsourcing?-A Case Survey. IEEE Transactions on Software Engineering (2017).

[29] Inc. PitchBook Data. 2015. European Middle Market Report 2H 2015. (2015).

[30] Inc. PitchBook Data. 2015. U.S. Middle market report Q4 2015. Technical Report.

[31] Randall W Rice, CSTE CSQA, and LLC Rice Consulting Solutions. 2003. Surviving the top ten challenges of software test automation. CrossTalk: The fournal of Defense Software Engineering (2003), 26-29.

[32] Per Runeson, Martin Höst, Austen Rainer, and Björn Regnell. 2012. Case study research in software engineering. John Wiley \& Sons, Inc., Hoboken, NJ, USA

[33] Bran Selic. 2009. Agile documentation, anyone? IEEE software 26, 6 (2009).

[34] Dag I K Sjoberg, Aiko Yamashita, Bente C D Anda, Audris Mockus, and Tore Dyba. 2013. Quantifying the effect of code smells on maintenance effort. IEEE Transactions on Software Engineering 39, 8 (2013), 1144-1156.

[35] Bradley R Staats, Katherine L Milkman, and Craig R Fox. 2012. The team scaling fallacy: Underestimating the declining efficiency of larger teams. Organizational Behavior and Human Decision Processes 118, 2 (2012), 132-142.

[36] Christoph Johann Stettina and Egbert Kroon. 2013. Is there an agile handover? an empirical study of documentation and project handover practices across agile software teams. In Engineering, Technology and Innovation (ICE) \& IEEE International Technology Management Conference, 2013 International Conference on. IEEE, 1-12.

[37] Stanley M Sutton, E C Cubed, and Mario Andretti. 2000. The Role of Process in a Software Start-up. IEEE Software 17, 4 (2000), 33-39.

[38] Henri Terho, Sampo Suonsyrjä, Aleksi Karisalo, and Tommi Mikkonen. 2015. Ways to cross the rubicon: pivoting in software startups. In International Conference on Product-Focused Software Process Improvement. Springer, 555-568.

[39] Edith Tom, Aybüke Aurum, and Richard Vidgen. 2013. An exploration of technical debt. Fournal of Systems and Software 86, 6 (jun 2013), 1498-1516.

[40] Je Voas, Ridgetop Circle, Lora Kassab, and College William. [n. d.]. Using Assertions to Make Untestable Software More Testable Keywords 1 Introduction. 1 ([n. d.]), 1-16. 